\title{
Studies of a Weakly Relativistic Oversized Backward Wave Oscillator with Disk Cathode and Rectangular Corrugation
}

\author{
Kazuo Ogura Member (Niigata University) \\ Yushi Miyazawa Non-member (Niigata University) \\ Satoshi Aoyama Non-member (Niigata University) \\ Yuki Takamura Non-member (Niigata University) \\ Syusuke Tamura Non-member (Niigata University) \\ Akira Sugawara Member (Niigata University)
}

Keywords : oversized backward wave oscillator, weakly relativistic, disk cathode, annular electron beam, rectangular corrugation

An oversized BWO with a novel cathode and a rectangular corrugation is studied. The beam voltage is weakly relativistic, less than $100 \mathrm{kV}$. For high-power microwave sources operating in relativistic regions, high-current relativistic electron beams are obtained by using cold cathodes, with the help of explosive emission. It is very difficult to realize a uniform electron beam. The cathode plays a very important role and has been studied extensively. To increase the azimuthal uniformity, the annular cathode surface needs to be modified, for example, coatings with velvet, carbon fiber or a mixture of fine graphite powder and epoxy. As the cathode voltage becomes lower, the uniform electron emission becomes the much more serious problem. To the best of our knowledge, velvet cathode is only one, which has been successfully used to generate uniform annular beam in the weakly relativistic. Velvet cathode can be used with the low-electric field, in return for the low-repetition rate and the short lifetime. For practical devises, it is necessary to develop a new cathode with a high-repletion and long lifetime, which can generate the uniform beam with a high current density even in the weakly relativistic region.

Sinusoidally corrugated periodic waveguides have been used in many high-power BWO experiments, since the electric fields become strong for high-power operations. For rectangularly corrugated SWSs, there exist strong electric fields near the sharp edges. The electric fields become unacceptably strong if used in high-power operations at power level above some hundreds of MW. However, for moderate power level of a few MW or less, the rectangular shape may be better than the sinusoidal shape, since it provides enough slowing of wave and adequate beam coupling.

This work is aimed at studying a weakly relativistic oversized BWO with a new cold cathode. The beam voltage is weakly relativistic, less than $100 \mathrm{kV}$. The beam current is up to about 500 A.

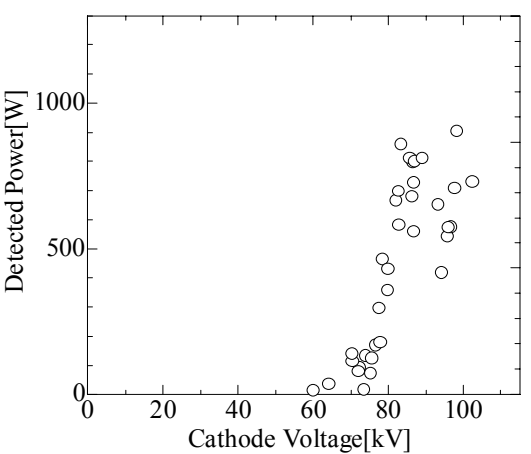

Fig. 1. Output radiation of oversized BWO versus the cathode voltage

We propose to use a novel disk cathode, in order to get a uniformly distributed annular electron beam. The novel disk cathode is successfully used even in the weakly relativistic region. This cold cathode is made of metal only and is a unique explosive cathode. By injecting the annular electron beam by the disk cathode, a weakly relativistic oversized BWO consisting of rectangularly corrugated cylindrical waveguide is demonstrated. The oscillation starting energy is about $60 \mathrm{kV}$ for a 10-period rectangular corrugation. As shown in Fig.1, the output power increases with increasing the beam voltage. The estimated output power reaches at about $200 \mathrm{~kW}$.

The mode control of the oversized BWO is a serious issue to be solved. The mode control between axisymmetric and nonaxisymmetric modes is performed by changing the end condition of BWO. The weakly relativistic oversized BWO mentioned above is unique and of considerable interest for practical use. 


\section{Studies of a Weakly Relativistic Oversized Backward Wave Oscillator with Disk Cathode and Rectangular Corrugation}

$\begin{array}{ll}\text { Kazuo Ogura* } & \text { Member } \\ \text { Yushi Miyazawa* } & \text { Non-member } \\ \text { Satoshi Aoyama* }^{*} & \text { Non-member } \\ \text { Yuki Takamura* } & \text { Non-member } \\ \text { Syusuke Tamura* } & \text { Non-member } \\ \text { Akira Sugawara* } & \text { Member }\end{array}$

Studies of an oversized BWO with a novel cathode and a rectangular corrugation are presented. The beam voltage is weakly relativistic, less than $100 \mathrm{kV}$. We propose to use a disk cathode made of metal only. The disk cathode can generate a uniformly distributed annular electron beam even in the weakly relativistic case. By using the annular electron beam, a weakly relativistic oversized BWO consisting of rectangularly corrugated cylindrical waveguide is demonstrated. The oscillation starting energy is about $60 \mathrm{kV}$ for a 10-period rectangular corrugation. The maximum output power is estimated about $200 \mathrm{~kW}$. The operation mode between axisymmetric and nonaxisymmetric modes can be controlled by changing the end condition of BWO.

Keywords : oversized backward wave oscillator, weakly relativistic, disk cathode, annular electron beam, rectangular corrugation

\section{Introduction}

Microwaves at moderate-power level or high-power level are demanded for widespread applications such as plasma heating, plasma diagnostics, telecommunication systems and radar systems. Slow-wave high-power microwave devices such as backward wave oscillator (BWO) can be driven by an axially injected electron beam without initial perpendicular velocity and has been studied extensively as a candidate for high power microwave sources $^{(1)}$. In the slow-wave devices, a slow-wave structure (SWS) is used to reduce the phase velocity of electromagnetic wave to the beam velocity. To increase the power handling capability and/or the operating frequency, oversized SWSs have been used successfully ${ }^{(2)-(9)}$. The term "oversized" means that the diameter is larger than free-space wavelength $\lambda$ of output electromagnetic wave by several times or more. The relativistic diffraction generator and multi-wave Cherenkov generator are special versions of the oversized BWO and have produced peak powers of GW level ${ }^{(2)(3)}$. For these relativistic devices, the pulsed power and magnetic field systems are very large and heavy. For practical applications, operations at reduced voltage and at reduced magnetic field are preferable, since the systems become compact. However, the phase velocity of electromagnetic mode should be slowed down close to the beam velocity, ensuring enough beam coupling with electromagnetic modes. This issue becomes very difficult by reducing the beam voltage. In Ref.(5), the power level about $500 \mathrm{MW}$ has been demonstrated at $8.3 \mathrm{GHz}$ (X-band) at a moderate voltage of about $500 \mathrm{kV}$.

In Refs.(4) and (6)-(9), we have been studying oversized BWOs operating in the relatively high frequency region, in K-band and Q-band. Unique features of our BWOs are (1) they are driven by a

* Graduate School of Science and Technology, Niigata University 8050 Igarashi 2-Nocho, Nishi-ku, Niigata 950-2181 weakly relativistic electron beam less than $100 \mathrm{kV}$, (2) the operation frequencies are relatively high, above $10 \mathrm{GHz}$ and (3) the guiding magnetic field is relatively low, less than $1 \mathrm{~T}$. Note that high-power operations beyond $10 \mathrm{GHz}$ are difficult for the conventional non-oversized slow-wave devices. Recently, the performance of weakly relativistic oversized BWO has been improved. Radiation powers up to about $500 \mathrm{~kW}$ (K-band) and up to about $200 \mathrm{~kW}$ (Q-band) have been demonstrated in Ref.(8).

For high-power microwave sources operating in relativistic regions (above a few hundred $\mathrm{kV}$ ), high-current relativistic electron beams are obtained by using cold cathodes, with the help of explosive emission. It is very difficult to realize a uniform electron beam. The cathode plays a very important role and has been studied extensively, see for example Chapter 9 of Ref.(1). To increase the azimuthal uniformity, the annular cathode surface needs to be modified, for example, coatings with velvet, carbon fiber or a mixture of fine graphite powder and epoxy. As the cathode voltage becomes lower, the uniform electron emission becomes the much more serious problem. To the best of our knowledge, velvet cathode is only one, which has been successfully used to generate uniform annular beam in the weakly relativistic $\mathrm{BWO}^{(4)(6)-(9)}$. Velvet cathode can be used with the low-electric field, in return for the low-repetition rate and the short lifetime. For practical devises, it is necessary to develop a new cathode with a high-repletion and long lifetime, which can generate the uniform beam with a high current density even in the weakly relativistic region.

Periodic structures are widely used in modern science and technology such as optics, acoustic design, infrared spectroscopy and microwave technology ${ }^{(10)}$. Sinusoidally corrugated periodic waveguides have been used in many high-power BWO experiments, since the electric fields become strong for high-power operations. For rectangularly corrugated SWSs, there 
exist strong electric fields near the sharp edges. The electric fields become unacceptably strong if used in high-power operations at power level above some hundreds of $\mathrm{MW}^{(5)}$. However, for moderate power level of a few MW or less, the rectangular shape may be better than the sinusoidal shape, since it provides enough slowing of wave and adequate beam coupling.

This work is aimed at studying a weakly relativistic oversized BWO with a new cold cathode. We propose to use a novel disk cathode shown in Fig.1(a), in order to get a uniformly distributed annular electron beam. The idea of disk cathode was presented in Ref.(11), in order to generate a long-pulse, intense relativistic electron beam. In Ref.(12), the disk cathode was used to avoid the pulse shortening in a non-oversized relativistic BWO and to study a long-pulse operation. In this paper, it is demonstrated that the novel disk cathode is successfully used even in the weakly relativistic region. This cold cathode is made of metal only and is a unique explosive cathode. By injecting the annular electron beam by the disk cathode, a weakly relativistic oversized BWO consisting of rectangularly corrugated cylindrical waveguide is demonstrated. Starting energy and operation mode of the oversized BWO are examined experimentally.

\section{Cold Cathode}

We use a cold cathode to obtain a beam with a high current density in the range of $100 \mathrm{~A} / \mathrm{cm}^{2}$ or more. It is very difficult to generate a uniformly distributed annular beam by the cold cathode, especially in the weakly relativistic region. In the weakly relativistic BWO, cathode voltage is less than $100 \mathrm{kV}$. The average electric field between the cathode and anode is about $10^{5} / \mathrm{cm}$ or less. In the past, we have used a hollow cathode with velvet on the axisymmetric emitting edge, in order to obtain an annular electron beam, see Fig.1(b) and Fig.2(b). The velvet cathode is the only cold cathode which operates properly in the weakly relativistic region, as in Refs.(4) and (6)-(9). The beam shape is observed by the burn patterns in thermally sensitive paper. By controlling velvet, the quality of beam by the hollow cathode is improved. A relatively uniform annular shape is realized as shown in Fig.3. The average radius of annular is nearly the same as the cathode diameter.

We propose to use a novel disk cathode in the weakly relativistic region less than $100 \mathrm{kV}$. The idea of disk cathode was presented by Loza et al. ${ }^{(11)}$, and used in the relativistic cases. In this paper, the novel cathode as shown in Fig.1(a) and 2(a) is tested in the weakly relativistic region. The burn patterns are shown in Fig.3. For a hollow cathode, the diameters of cathode and beam limiter are $23 \mathrm{~mm}$ and $26 \mathrm{~mm}$, respectively. For a disk cathode, the diameters of cathode and beam limiter are $25 \mathrm{~mm}$ and $28 \mathrm{~mm}$, respectively. The beam generated by the disk cathode has the more uniform distribution and has the higher current density compared with the hollow cathode with velvet. Note that any coating on the disk cathode surface is not required. This is a surprising result. It is clear that the disk cathode is suitable for the weakly relativistic case as well as the relativistic case. As can be seen from Fig.1, the novel disk cathode is expected to reduce the unwanted plasma produced at the cathode and paves the way for long-pulse high-power microwave sources.

\section{Rectangular SWS}

Cylindrical SWS is corrugated periodically as shown in Fig.4. Dispersion characteristics of SWS are determined by average
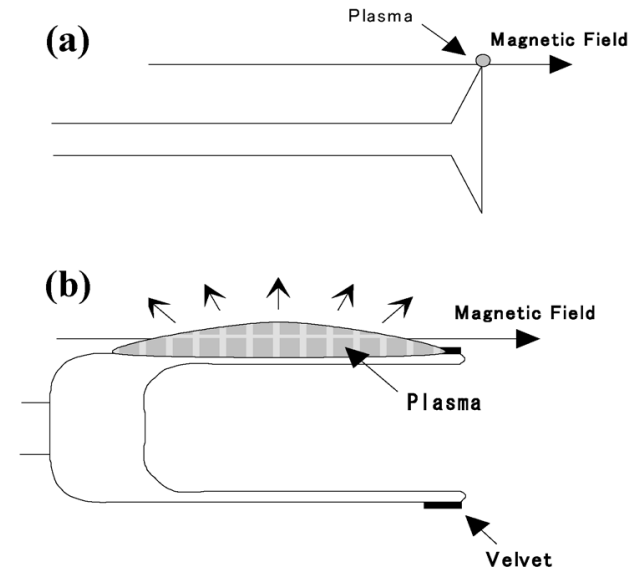

Fig. 1. Cross sections of (a) disk cathode and (b) hollow cathode
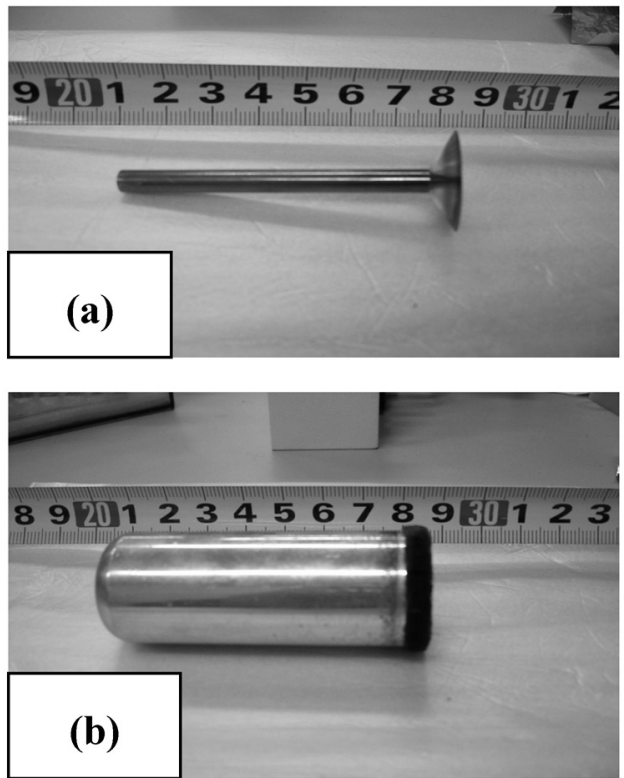

Fig. 2. Photographs of (a) disk cathode and (b) hollow cathode. The disk cathode is made of copper; The hollow cathode is made of aluminum with velvet on the edge
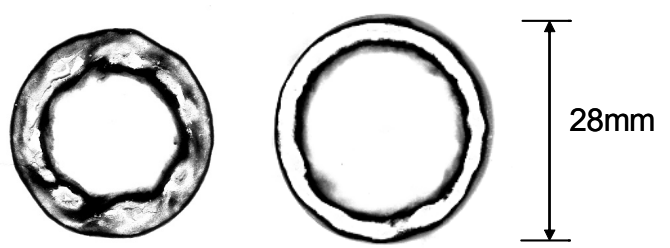

Fig. 3. The burn patterns of annular electron beam. Left-side is for a hollow cathode with velvet obtained from five-shot overlay at about $90 \mathrm{kV}$; Right-side is for a novel disk cathode obtained from one-shot at about $80 \mathrm{kV}$

radius $R_{0}$, corrugation amplitude $h$, corrugation width $d$ and periodic length $z_{0}$. The corrugation wave number is given by $k_{0}=2 \pi / z_{0}$. The dispersion characteristics of the structure are controlled by changing $R_{0}, h, d$ and $z_{0}$. Parameters of rectangular SWS are listed in Table 1. We also list parameters of sinusoidal 


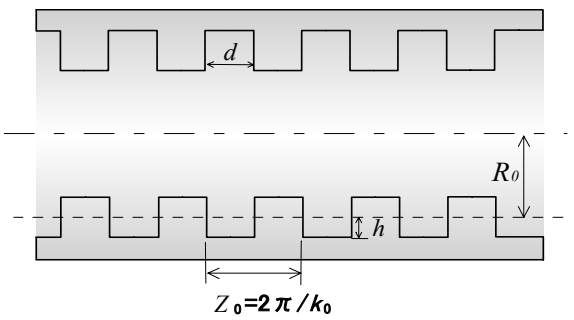

Fig. 4. Schematic diagram of rectangularly corrugated SWS

Table 1. Parameters of Periodically Corrugated SWS

\begin{tabular}{|c|c|c|c|c|}
\hline & $\begin{array}{c}R_{0} \\
{[\mathrm{~mm}]}\end{array}$ & $\begin{array}{c}h \\
{[\mathrm{~mm}]}\end{array}$ & $\begin{array}{c}d \\
{[\mathrm{~mm}]}\end{array}$ & $\begin{array}{c}z_{0} \\
{[\mathrm{~mm}]}\end{array}$ \\
\hline Rectangular SWS & 15.1 & 1.1 & 1.5 & 3.0 \\
\hline Sinusoidal SWS & 15.6 & 1.65 & --- & 3.0 \\
\hline
\end{tabular}

corrugation having almost the same dispersion characteristics as the rectangular SWS.

To analyze electromagnetic field properties of periodic system, various methods including the Rayleigh method and the field matching method have been developed ${ }^{(10)}$. The Rayleigh method is based on the Rayleigh hypothesis, which assumes that the fields inside the periodic corrugation are also expressed by the same spatial harmonic series as outside the corrugation. The shortcoming of the method is that it is difficult to obtain exact results in case of the deep corrugation. Theoretical limit for its applicability has been proposed and argued extensively. For the field matching method, there is no such limitation. In the field matching method, the field inside corrugation is expressed by appropriate functions so as to satisfy the boundary conditions at the wall of rectangular corrugation. Then, the functions inside corrugation are matched to the periodic functions outside corrugation, by using the boundary conditions at the boundary surface between the inside and outside the corrugation. The Rayleigh method is particularly simple and important for self-consistent analyses ${ }^{(13)-(15)}$. The method is suitable for analyses of sinusoidally corrugated SWS, which is used in many high-power BWO experiments. For the rectangular corrugation, the field properties are obtained by the field matching method. The dispersion curves of the lowest axisymmetric transverse magnetic $\left(\mathrm{TM}_{01}\right)$ mode are shown in Fig.5. The growth rate for the rectangular SWS is higher than that of the sinusoidal SWS. The rectangular shape provides strong beam coupling. It is better than the sinusoidal shape. As compared with the sinusoidal shape, the rectangular shape is easier to manufacture and the manufacture accuracy is better. Hence, the dispersion characteristics are controlled more easily and accurately.

As is discussed in Ref.(6), there exist two threshold conditions for the oscillation in the SWS, i.e., the starting current and the starting energy. In order to satisfy the latter condition, the interaction width $\Delta k_{\mathrm{z}}$ in the wave number space should be larger than $2 \pi / L$. Here, $L$ is the length of SWS. In the oversized SWS, this condition becomes critical and cannot be satisfied by increasing only the beam current. Figure 6 shows how $\Delta k_{\mathrm{z}}$ change with increasing beam energy. By increasing beam energy and approaching the beam interaction point to the $\pi$ point, the width $\Delta k_{\mathrm{z}}$ increases. At a critical beam energy (so-called starting energy), $\Delta k_{\mathrm{z}}$ becomes broad enough to satisfy the oscillation condition. Oscillations will not start up to $80 \mathrm{kV}$ for sinusoidal SWS with $L=$

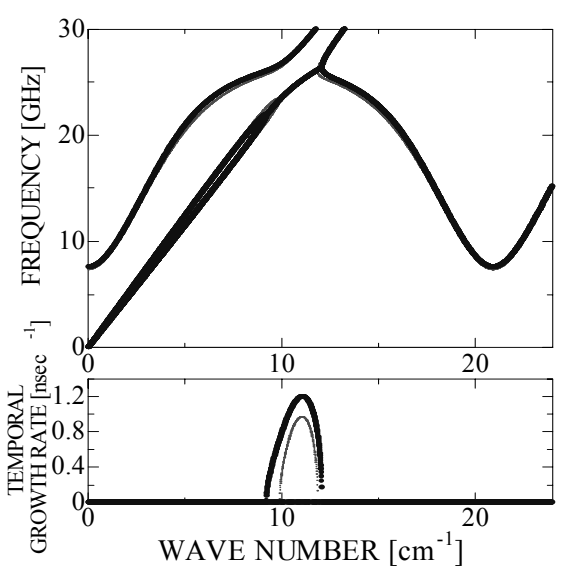

Fig. 5. Dispersion curves of fundamental $\mathrm{TM}_{01}$ for periodic SWS listed in Table 1; Thick line is for the rectangular SWS and thin line for the sinusoidal SWS

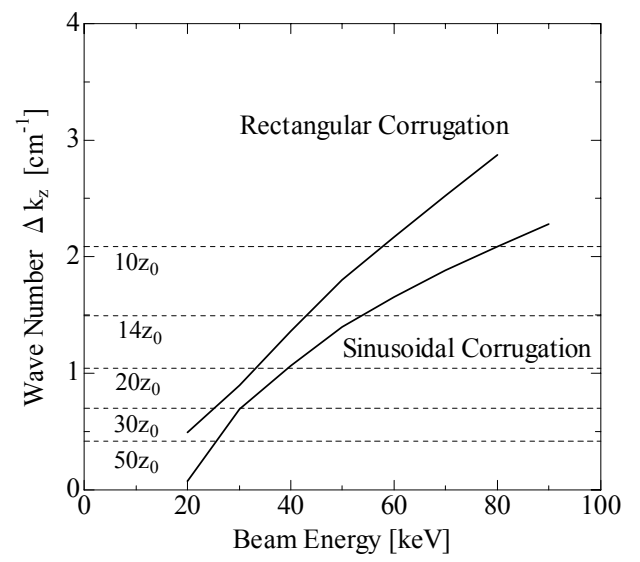

Fig. 6. Numerically obtained interaction width $\Delta k_{\mathrm{z}}$ versus beam energy; Horizontal dashed lines are $2 \pi / L$ for the SWS length $L$ from $10 z_{0}$ to $50 z_{0}$

$10 z_{0}$. For the rectangular SWS with $L=10 z_{0}$, it is predicted that the oscillation will start about $58 \mathrm{kV}$. For the sinusoidal SWS, the beam coupling becomes weak and the starting energy becomes higher compared with the rectangular case.

\section{Oversized BWO Experiment}

Experimental setup is schematically shown in Fig.7. Output voltage up to about $100 \mathrm{kV}$ from the pulse-forming line is applied to a cold cathode. A hollow cold cathode with velvet on the emitting edge is used. Uniform magnetic field $B_{0}$ is applied. The maximum value of $B_{0}$ is about $1 \mathrm{~T}$. The microwave output is picked up by a rectangular horn antenna located away from the output window.

In Fig. 8, an example of detected signal is shown. The beam voltage and current are respectively about $98 \mathrm{kV}$ and $470 \mathrm{~A}$, at the microwave peak. The microwave signal is split into two branches. One consists of a short waveguide and forms a prompt signal. The other is a delay line and forms a delayed signal. The delay time after about $31 \mathrm{~m}$ delay line is $152 \mathrm{nsec}$, from which the radiation frequency is estimated to be $26 \mathrm{GHz}$. The observed frequencies are fairly consistent with the frequencies of crossing points between the beam line and the fundamental $\mathrm{TM}_{01}$ of SWS.

There exist other fundamental modes such as nonaxisymmetric 


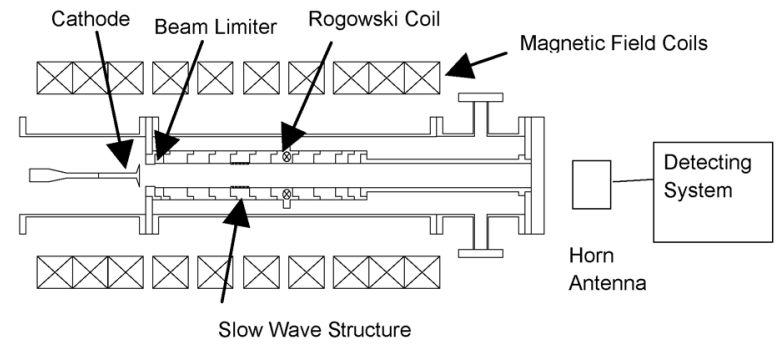

Fig. 7. Schematic diagram of experimental setup for the oversized BWO

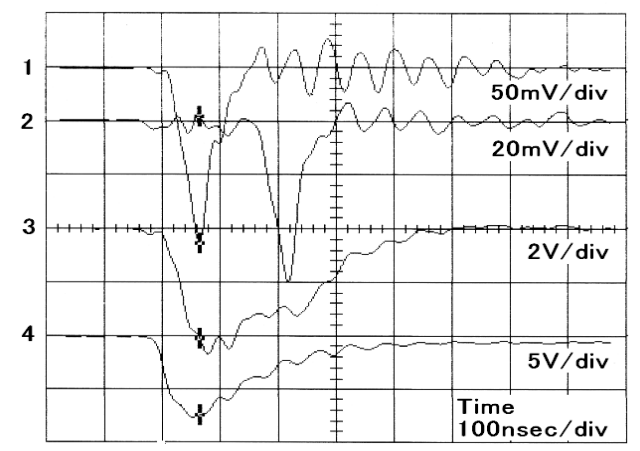

Fig. 8. Waveforms of measured signal:1 prompt signal, 2 delayed signal, 3 beam current and 4 beam voltage

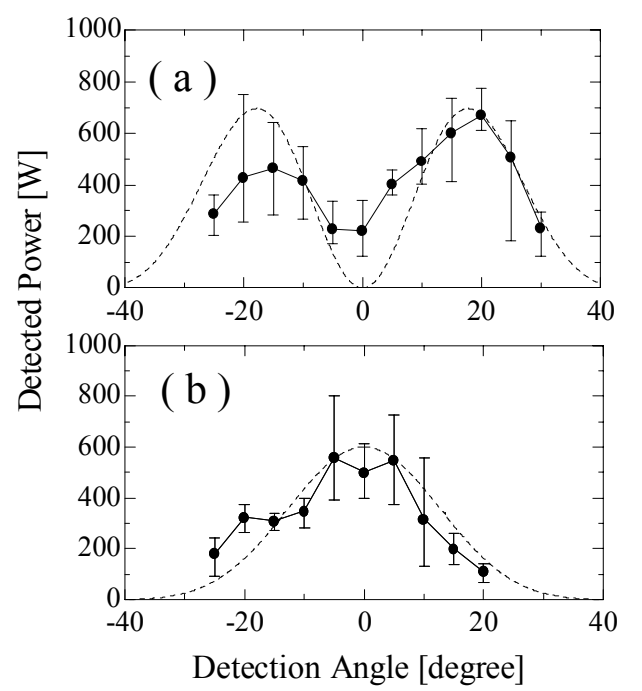

Fig. 9. Radiation patterns with the straight cylinder length of (a) $66 \mathrm{~mm}$ and (b) $100 \mathrm{~mm}$; Dashed curves are theoretical curves of (a) $\mathrm{TM}_{01}$ and (b) $\mathrm{HE}_{11}$ mode, respectively; The beam voltage is in the range of $85-100 \mathrm{kV}$ and error bar expresses scattering of data at each angle

hybrid $\mathrm{HE}_{11}$ and $\mathrm{HE}_{21}$ modes ${ }^{(7)(9)}$, very close to the fundamental $\mathrm{TM}_{01}$. The operation mode cannot be determined uniquely by the frequency measurement only. To examine the mode, the radiation patterns are measured as shown in Fig.9, by changing the detection angle in an equatorial plane around a pivot at the center of output window. The operation mode changes by the straight cylinder length before SWS in Fig.7. For a $66 \mathrm{~mm}$ length straight cylinder, the dominant radiation mode is $\mathrm{TM}_{01}$ mode as Fig.9(a). By increasing the length to $100 \mathrm{~mm}$, the radiation mode becomes

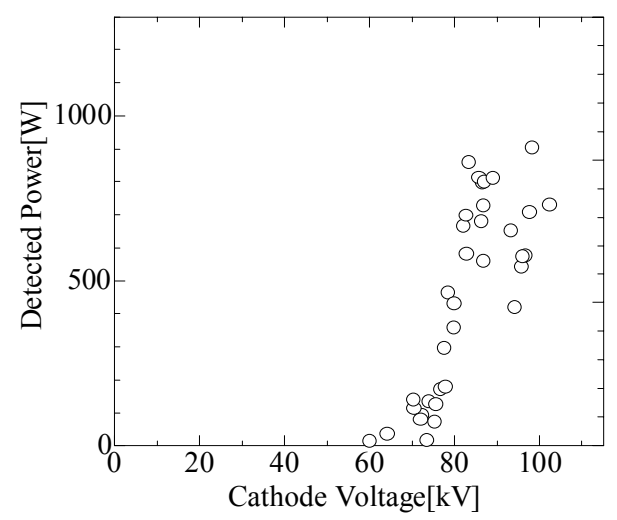

Fig. 10. Output radiation of oversized BWO versus the cathode voltage

$\mathrm{HE}_{11}$ mode as Fig.9(b). This mode predominantly consists of nonaxisymmetric transverse electric $\mathrm{TE}_{11}$ component and is characterized by a peak at the center. For the longer cylinder than $100 \mathrm{~mm}, \mathrm{TM}_{01}$ mode becomes dominant again. Both axisymmetric and nonaxisymmetric modes are observed and are controlled by the axial condition, i.e., the input cylinder length.

Figure 10 shows the dependence of the detected microwave power on the beam voltage. The operation mode is the fundamental $\mathrm{TM}_{01}$ mode. The weakly relativistic oversized BWO generates meaningful radiations above the starting energy for the rectangular SWS. Some peaks can be seen and may correspond to axial modes of the SWS cavity. The operating axial mode changes by changing the beam voltage. By increasing the beam voltage, the interacting point between the beam and electromagnetic wave is shifted to the point of $k_{\mathrm{z}} \mathrm{z}_{0}=\pi$. The maximum power is estimated to be about $200 \mathrm{~kW}$.

\section{Discussion and Summary}

In the relativistic high-power microwave devices, high-current electron beam are generated based on the explosive emission. In the explosive emission, plasma is generated on the cathode surface and acts as electron emitter. The cathode plasma expansion will close the cathode-anode gap and terminate the electron beam. This results in the microwave pulse shortening. For the hollow cathode, a large amount of unwanted plasma might be formed along the cathode surface as in Fig.1(b). A way of overcome this problem is disk cathode, as presented in Ref.(11). A disk cathode reduces the amount of unwanted plasma. It was also reported that the angular spectrum of electrons is small enough to apply high-power microwave devices. The disk cathode is a promising candidate for a useful long-pulse explosive cathode. However, it was considered for the relativistic devises and the operation in the weakly relativistic region was not expected. In this paper, we demonstrate that an explosive cathode made of metal only can be available even for the weakly relativistic case. Material such as aluminum, copper and stainless steel are tested as cathode. Up to now, the best result is obtained by a copper cathode. It is also expected that shape of emitting edge and surface condition have a strong effect on the performance. More definite study on the emission mechanism should be made in the future.

In the high-power operation of the oversized BWO, the beam interaction with the nonaxisymmetric mode is the same as the axisymmetric interaction. For the high-power operation of weakly 
relativistic oversized BWO based on the sinusoidal SWS in Ref.(9), the nonaxisymmetric mode became dominant mode. The mode control of the oversized BWO is a serious issue to be solved. In this work, the nonaxisymmetric and axisymmetric operations of the oversized BWO are observed. It is demonstrated that these modes can be controlled by changing the input cylinder length.

In conclusion, we study an oversized BWO with a novel disk cathode and a rectangular corrugation. The beam voltage is weakly relativistic, less than $100 \mathrm{kV}$. The beam current is up to about $500 \mathrm{~A}$. In order to get a uniformly distributed annular electron beam, we propose a disk cathode made of metal. The novel cathode is able to be used even in the weakly relativistic case. By using the annular electron beam, a weakly relativistic oversized BWO consisting of rectangularly corrugated cylindrical waveguide is demonstrated. The oscillation starting energy is about $60 \mathrm{kV}$ for a 10-period rectangular corrugation. The output power increases with increasing the beam voltage, if the beam interaction point approaches to the $\pi$ point. The estimated output power reaches at about $200 \mathrm{~kW}$. The mode control between axisymmetric and nonaxisymmetric modes is performed by changing the end condition of BWO. The weakly relativistic oversized BWO mentioned above is unique and of considerable interest for practical use.

\section{Acknowledgements}

This work was partially supported by Grant-in-Aid for Scientific Research from the Ministry of Education, Science, Sports and Culture of Japan, and by Collaborative Research Program between NIFS and University of Tsukuba in cooperation with Japanese universities.

(Manuscript received Dec. 25, 2006, revised March 22, 2007)
(9) K. Ogura, Y. Miyazawa, Y. Kiuchi, S. Aoyama, H. Tanaka, and A. Sugawara : "Operation Mode of Oversized Backward Wave Oscillator Driven by Weakly Relativistic Electron Beam", The 3rd Japan-US Symposium on Pulsed Power and Plasma Applications, PST-06-59/PPT-06-42, Kauai, USA (2006)

(10) E. G. Loewen and E. Popov : Difraction Gratings and Applications, Marcel Dekker, New York (1997)

(11) O. T. Loza and I. E. Ivanov : "Measurement of the Angular Spectrum of Electrons in a High-Current Magnetized REB with Microsecond Duration", Proc. 13th Int. Conf. High-Power Particle Beams, pp.603-606, Nagaoka, Japan (2000)

(12) K. Han, M. I. Fuks, and E. Schamiloglu : "Initial Studies of a Long-Pulse Relativistic Backward-Wave Oscillator Utilizing a Disk Cathode", IEEE Trans. Plasma Sci., Vol.30, pp.1112-119 (2002)

(13) O. Watanabe, K. Ogura, T. Cho, and Md. R. Amin : "Self-Consistent Linear Analysis of Slow Cyclotron and Cherenkov Instabilities", Phys. Rev. E, Vol.63, p.6503(1-9) (2001)

(14) H. Yamazaki, O. Watanabe, Y. Yamashita, M. Iwai, Y. Suzuki, and K. Ogura : "Cherenkov and Slow Cyclotron Instability in Periodically Corrugated Cylindrical Waveguide in Low Magnetic Field Region", IEEJ Trans. FM, Vol.124, No.6, pp.477-482 (2004)

(15) Y. Kiuchi, H. Tanaka, K. Ogura, O. Watanabe, T. Cho, and Md. R. Amin : "Slow Cyclotron and Cherenkov Instabilities in Weakly Relativistic Oversized Backward Wave Oscillator", Trans. Fusion Sci. Tech., Vol.51, pp.331-333 (2007)
Kazuo Ogura

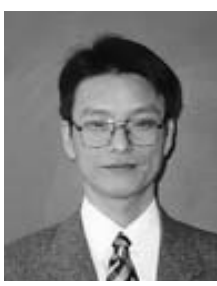

Yushi Miyazawa

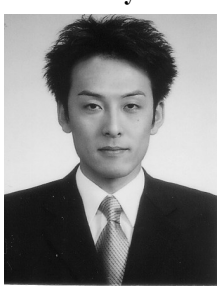

Satoshi Aoyama pp.525-536 (1990)

(4) K. Ogura, M. R. Amin, K. Minami, X. D. Zheng, Y. Suzuki, W. S. Kim, T. Watanabe, Y. Carmel, and V. L. Granatstein : "Experimental Demonstration of a High-Power Slow-Wave Electron Cyclotron Maser Based on a Combined Resonance of Cherenkov and Anomalous Doppler Interactions", Phys. Rev. E, Vol.53, pp.2726-2729 (1996)

(5) A. N. Vlasov, A. G. Shkvarunets, J. C. Rodgers, Y. Carmel, T. M. Antonsen, Jr., T. M. Abuelfadl, D. Lingze, V. A. Cherepenin, G. S. Nusinovich, M. Botton, and V. L. Granatstein : "Overmoded GW-Class Surface-Wave Microwave Oscillator”, IEEE Trans. Plasma Sci., Vol.28, pp.550-560 (2000)

(6) K. Ogura, R. Yoshida, Y. Yamashita, H. Yamazaki, K. Komiyama, and M. Sakai : "Study on Oscillation Starting Condition of K-Band Oversized Backward Wave Oscillator Driven by a Weakly Relativistic Electron Beam", J. Plasma Fusion Res. SERIES, Vol.6, pp.703-706 (2004)

(7) K. Ogura, K. Komiyama, M. Sakai, D. Yamada, H. Saito, and H. Yamazaki : "Performance of Weakly Relativistic Oversized Backward Wave Oscillators", IEEJ Trans. FM, Vol.125, No.9, pp.733-738 (2005)

(8) S. Aoyama, Y. Miyazawa, K. Ogura, A. Sugawara, and M. Hirata : "Improved Performance of Oversized Backward Wave Oscillator driven by Weakly Relativistic Electron Beam", Trans. Fusion Sci. Tech., Vol.51, pp.325-327 (2007)

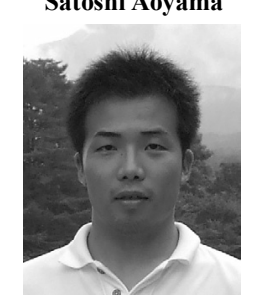

Yuki Takamura

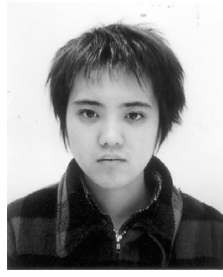

(Non-member) was born in Japan in 1982.He received the B.S. and M.S degrees in engineering from Niigata University, Japan, respectively 2005 and 2007. He has been working at Aisin Seiki Co. Ltd., since 2007.

(Member) was born in Japan in 1957. He received the B.S. degree in physic from Okayama University in 1981, and the M.S. and Ph.D degrees in physics from Kyoto University in 1983 and 1989, respectively. He is a Professor in the Electrical and Electronic Engineering Department of Niigata University. His research interests include high-power microwave devices and their applications to plasma heating and control.

(Non-member) was born in Japan in 1982.He received the B.S. and M.S degrees in engineering from Niigata University, Japan, respectively in 2005 and 2007. He has been working at Tokyo Electric Co. Ltd., since 2007. 
Syusuke Tamura

(Non-member) was born in Japan in 1984. He received the B.S. degree from Niigata University in March 2006. $\mathrm{He}$ is currently a graduate student in the Graduate School at the same university.
Akira Sugawara

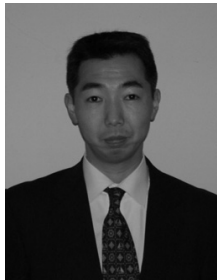

(Member) is Associate Professor at the Department of Electrical and Electronic Engineering, Faculty of Engineering, Niigata University. He received BE(1989) and ME(1991) and Ph.D. (1994) in Electrical Engineering from Niigata University. He joined Niigata University as Associate Professor in 1998, Lecturer (1997-1998), and Research Associate (1994-1997). He is engaged in research on TVGs and applications. He was Visiting Scientist, Massachusetts Institute of Technology, USA (2000-2001). $\mathrm{He}$ is a member of The Physical Society of Japan and The Japan Society of Plasma Science and Nuclear Fusion Research. 